
$\mathrm{XVI})$

\title{
Análisis distribucional de las estatuillas incaicas encontradas en el volcán Llullaillaco
}

Analyse distributionnelle des statuettes incas retrouvées dans le volcan Llullaillaco

Distributional analysis of the Inca statuettes found in Llullaillaco volcano

\section{Pablo Mignone}

\section{CpenEdition}

Journals

Edición electrónica

URL: http://journals.openedition.org/bifea/8145

DOI: 10.4000/bifea.8145

ISSN: 2076-5827

Editor

Institut Français d'Études Andines

Edición impresa

Fecha de publicación: 1 abril 2017

Paginación: 77-96

ISSN: 0303-7495

Referencia electrónica

Pablo Mignone, «Análisis distribucional de las estatuillas incaicas encontradas en el volcán Llullaillaco », Bulletin de l'Institut français d'études andines [En línea], 46 (1) | 2017, Publicado el 08 abril 2017, consultado el 05 noviembre 2020. URL : http://journals.openedition.org/bifea/8145; DOI : https://doi.org/10.4000/bifea.8145

\section{(c) () 8 ()}

Les contenus du Bulletin de l'Institut français d'études andines sont mis à disposition selon les termes de la licence Creative Commons Attribution - Pas d'Utilisation Commerciale - Pas de Modification 4.0 International. 


\title{
Análisis distribucional de las estatuillas incaicas encontradas en el volcán Llullaillaco
}

\author{
Pablo Mignone*
}

\begin{abstract}
Resumen
Hemos realizado, en esta ocasión, un estudio distribucional de las estatuillas antropomorfas y zoomorfas incaicas que fueron encontradas a $6715 \mathrm{msnm}$ en el volcán Llullaillaco (Salta-Argentina) junto a tres cuerpos humanos. Estas ofrendas fueron enterradas en el marco de un ritual incaico conocido como capacocha, por el cual se sellaban y mantenían las alianzas entre las comunidades aborígenes y el Estado. Los resultados obtenidos muestran una distribución diferencial entre los cuerpos y las figurillas antropomorfas, formando grupos segregados sin relación espacial. Esto corroboraría arqueológicamente la información etnohistórica acerca de la importancia sui generis de las estatuillas ofrecidas, en muchas ocasiones sin acompañamiento de restos humanos. Finalmente, la distribución nos plantea incógnitas en cuanto a la secuencia de depositación de las ofrendas de capacocha del volcán Llullaillaco, sugiriendo la sincronía de los entierros o un relativo corto tiempo entre los mismos.
\end{abstract}

Palabras clave: inca, capacocha, Llullaillaco, estadística, distribución

\section{Analyse distributionnelle des statuettes incas retrouvées dans le volcan Llullaillaco}

\section{Résumé}

Nous avons réalisé, pour cette occasion, une analyse distributionnelle des statuettes anthropomorphes et zoomorphes incas, mise au jour à 6715 mètres d'altitude dans le volcan Llullaillaco (Salta-Argentine) avec trois corps humains. Ces offrandes ont été enterrées dans le cadre d'un rituel inca connu comme capacocha, par lequel étaient scellées et maintenues les alliances entre les communautés aborigènes et l'État. Les résultats obtenus montrent une distribution différentielle entre les corps et les figurines anthropomorphes, formant des groupes ségrégués sans relation spatiale. Ceci corroborerait sur le plan archéologique l'information ethnohistorique qui souligne l'importance sui generis des statuettes offertes

* Investigador CONICET, Instituto de Ciencias Sociales y Humanidades, Universidad Nacional de Salta, Argentina. E-mail: pmignone@hotmail.com 
à de nombreuses occasions sans accompagnement de restes humains. Enfin, la distribution laisse subsister des doutes quant à la séquence de dépôt des offrandes de capacocha du volcan Llullaillaco, suggérant la synchronie des enterrements ou un temps relativement court entre ces derniers.

Mots-clés : Inca, capacocha, Llullaillaco, statistique, distribution

\title{
Distributional analysis of the Inca statuettes found in Llullaillaco volcano
}

\begin{abstract}
We conducted a study of the distribution of anthropomorphic and zoomorphic inca figurines found in the tombs of three human bodies, at 6715 meters above sea level in the Llullaillaco (Salta-Argentina) volcano. These offerings were buried in an Inca ritual known as capacocha, by which were sealed and maintained partnerships between aboriginal communities and the state. The results of our study show a differential distribution between the bodies and anthropomorphic figurines, forming groups segregated without spatial relationship. This corroborates the ethnohistorical and archaeological information about the importance of figurines offered on many occasions without accompanying human remains. Finally, the distribution raises uncertainties as to the sequence of deposition of the offerings of the Llullaillaco volcano, suggesting the synchronicity of burials or a relatively short time between them.
\end{abstract}

Keywords: Inca, capacocha, Llullaillaco, statistics, distribution

\section{INTRODUCCIÓN}

En 1999 se hallaron los restos de una ofrenda ritual incaica llamada capacocha, a 6715 msnm, cerca de la cima del volcán Llullaillaco, una de las montañas más altas del noroeste argentino.

Se encontraron, a casi $2 \mathrm{~m}$ bajo tierra, tres cuerpos humanos (dos niñas y un niño, de 6,15 y 7 años, respectivamente), junto a más de cien objetos (Reinhard \& Ceruti, 2010).

Aunque hasta el desarrollo de nuestro trabajo no se habían realizado análisis de distribución de los objetos y cuerpos en los espacios mortuorios que revelen probables patrones de asociación, las interpretaciones sobre este contexto arqueológico se centran en las características tipológicas de los objetos para subrayar el carácter propiciatorio de las ofrendas y la voluntad incaica de representar en un espacio mortuorio el microcosmos del Estado y de hacer una demostración de su poder (Reinard \& Ceruti, 2000; 2010; Ceruti, 2003; Besom, 2009; entre otros).

Como aporte a estos antecedentes, buscamos mostrar una nueva mirada sobre las ofrendas incaicas, con base en la distribución de los hallazgos, identificando grupos de ofrenda y relaciones entre los mismos a través de un estudio estadístico que se basa en la descripción publicada de los contextos de hallazgo (Reinhard \& 
Ceruti, 2000; 2010: apéndice B), la cual transformamos en una matriz X, Y, Z para estudiar la distancia y la profundidad de la evidencia.

Las pruebas estadísticas no paramétricas muestran que los hallazgos se dividen entre «lo masculino»y «lo femenino», según su especificidad sexual.

Por otro lado, los resultados indican que no hay relación espacial entre los objetos suntuarios incaicos y los cuerpos humanos. Esto sugiere, entonces, que son el producto de distintos momentos de inhumación (afirmación temeraria en ausencia de estudios estratigráficos detallados) o de distintas lógicas de distribución de objetos por parte de los oferentes.

\section{LAS MINIATURAS HUMANAS Y DE LOS RECURSOS NATURALES EN EL MUNDO ANDINO}

Los objetos presentados en este artículo han sido interpretados (por Reinhard \& Ceruti, 2000; 2010; Ceruti, 2003; entre otros) como pertenecientes a uno de los cinco tipos de sacrificios humanos predominantes entre los incas: la capacocha (Besom, 2009: 401), que era una forma de tributo que el Inca exigía a las provincias y que recaía en niños (cochaguas) y jóvenes mujeres tomadas de las casas de escogidas (acllahuasi) (Cobo, 1964 [1653]: 134; Pizarro, 1986 [1571]).

La capacocha tenía como fin político integrar simbólicamente y delinear los territorios y los límites de las poblaciones sujetas al poder estatal (Zuidema, 1973; Duviols, 1976; MacCormack, 2000; Mc Ewan \& Van de Guchte, 1993), ya que muchos de los niños ofrecidos eran hijos e hijas de curacas provinciales (Betanzos, 1987 [1551-1557]), que obtenían a cambio de ellos cargos políticos de mano del soberano (Hernández Príncipe, 1923 [1622]: 63).

Junto a los cuerpos humanos, y a veces en sustitución de ellos (Schobinger, 1999: 21), se ofrecían figurillas antropomorfas y zoomorfas.

Las miniaturas antropomorfas fueron interpretadas por los mismos cronistas como la representación de las deidades (Murúa, 1946 [1590]: 257; Cobo, 1964 [1653]: 46; Betanzos, 1987 [1551-1557]: 110), de los sirvientes de las deidades residentes en las montañas (Arriaga, 1968 [1621]: 11 y 86), o bien de la élite real incaica (Betanzos, 1987 [1551-1557]: 48; Polo de Ondegardo, 1916 [1571]: 194).

Las miniaturas antropomorfas femeninas, usualmente de plata, son entendidas como ofrendas sustitutivas de mujeres (Schobinger, 1999: 21) o la representación simbólica de vírgenes escogidas, o acllas (Farrington, 1998: 56). Algunos autores sostienen que dichas figurillas representan a la Mama Quilla, la Luna (Beorchia Nigris, 1985: 393), mientras que otros afirman que representan a la Pachamama, la tierra (Rebitsch, 1957: 202).

Por su parte, las miniaturas masculinas, principalmente de oro, manifiestan algunos atributos característicos de la nobleza incaica, como las orejas perforadas y alargadas (Reinhard \& Ceruti, 2010: 139) y un bulto en una de sus mejillas que indica el consumo de coca (Dransart, 1995: 5; Salazar \& Burger, 2004: 198). 
Por último, Reinhard \& Ceruti (2010: 142) sostienen que las miniaturas del Llullaillaco no son sacrificios sustitutivos, ya que fueron enterradas junto a las ofrendas humanas y no en lugar de ellas. Es más probable, argumentan los autores, que en la mayoría de los casos las estatuillas masculinas y femeninas representaran a las deidades asociadas con el volcán y otras montañas de la región, al igual que las deidades principales de la cosmología incaica, como el Sol (Inti) y el clima (Illapa).

Existe consenso al afirmar que estas figurillas representaban, a su vez, el universo social de los adultos, la ideología imperial, la jerarquía estatal, los súbditos y, en el caso de las miniaturas zoomorfas, las posesiones imperiales y los anhelos expansivos (D'Altroy, 2003; Mc Ewan \& Van de Guchte, 1993; Schobinger, 2001), y que fueron empleadas como un medio religioso para propiciar la reproducción del rebaño (Duviols, 1986: 285; Farrington, 1998: 56; Mills, 1997: 94). En algunos contextos rituales, este tipo de estatuillas podrían haber actuado también como sustituto de los animales representados (Farrington, 1998: 56; Schobinger, 1999: 21).

El uso de miniaturas en los Andes no es exclusivo de los incas y forma parte de una larga tradición que ha sido registrada arqueológicamente en culturas tales como Paracas, Nazca, Moche y Tiwanaku. Se puede observar todavía, después de la conquista española, a través de distintos tipos de fuentes documentales y registros etnohistóricos y etnográficos (López, 2012: 48).

En la arqueología del extremo sur del mundo andino, en los actuales territorios de Chile y Argentina, también se pueden encontrar ejemplos de objetos en miniatura y representaciones rupestres, en particular de camélidos, los que poseían probablemente una función mágica analógica, asociados generalmente a la fertilidad y a la abundancia (López, 2012: 52).

Las figuras modeladas en diversos materiales, como madera, piedra, metal y cerámica, representan tanto a seres humanos como a recursos económicos: cultígenos (maíz, papa) y animales (llamas, alpacas y vicuñas).

Estas figuras responden a la categoría genérica de huaca, es decir, cualquier elemento físico, natural o artificialmente construido, espíritu humano o animal, fuerza de la naturaleza o vitalidad humana, considerado sagrado y al cual se le rinde culto (Manzo, 2008: 147).

No son los elementos en sí los adorados, sino la fuerza inmaterial contenida en ellos, el enqa, que habitaba por igual en los cerros o en los lagos, en las lagunas o en los manantiales (Manzo, 2008: 147).

En diversos contextos modernos, las conopas, piedras que poseen forma de animales o que son de colores y formas consideradas extrañas, son sagradas y se les emplea en ceremonias relacionadas con el éxito de las actividades socioeconómicas: siembra y cosecha de plantas, carneada, señalada y venta de animales y ritos de multiplicación de rebaños.

De acuerdo con la actividad económica implicada, adquieren diferentes denominaciones: zarapconopa para el maíz, papaconopa para la papa, 
llamaconopa o caullama para la llama. Dentro de estas últimas, se encuentran las illas, un tipo especial de conopa que representa de forma exclusiva figuras de animales domésticos que tienen un valor sagrado por intervenir en ritos o ceremonias, ejecutados para propiciar el aumento y prosperidad del rebaño (Manzo \& Raviña, 1996).

El término illa fue definido en el siglo XVII por González Holguín, como «La piedra vezar grande, o notable como vn hueuo, o mayor, que la trayan consigo por abusion para ser ricos y venturosos»; su portador, «Yllayoc runa», «es el hombre muy rico y venturoso que tiene y guarda tesoro»; quien se enriquece rápidamente, es llamado «Yllayoc», y la casa rica y abundante, «Ylla huaci» (González Holguín, 1989 [1613]: 366-367). Illa en aimara, según Bertonio, significa un objeto valioso, «...Qualquier cosa que vno guarda para provision de su casa, como Chuňo, Mayz, Plata, Ropa, y aun las joyas... Piedra bazzar grande que se halla dentro de las vicunas, o carneros (Bertonio en Flores Ochoa, 1974: 252).

Otras referencias indican que mientras las illas son de carácter público - y comunes a toda una provincia, un pueblo o ayllu-, las conopas son secretas y familiares o particulares, representaciones de dioses domésticos que pertenecieron a los habitantes de cada casa (Manzo, 2008: 159).

Flores Ochoa (1974), por su parte, con base en sus trabajos etnográficos en comunidades pastoriles de Puno, diferencia illa, conopa y enqaychu.

Según este autor, las illa son pequeñas esculturas de piedra (por lo general de cuarcita, basalto, granito u otras piedras de grano fino) que representan a las alpacas, llamas u ovejas. Algunas illas son piedras de formas naturales que asemejan animales, o bien esculturas magníficamente hechas con orificios en la parte superior del lomo llamados qocha o laguna. Los enqaychu, por su parte, son piedras naturales, de no más de ocho centímetros de largo, o bien las piedras bezoares halladas en las vísceras de las llamas, alpacas, vicuñas y tarucas (Flores Ochoa, 1974: 249).

De igual forma, en las comunidades pastoriles de Cerrillos (provincia de Sud Lípez, departamento de Potosí, Bolivia), las figuras de camélidos en piedra forman parte importante de la vida ritual y económica. Ellas adquieren un singular valor con los viajes de intercambio que realizan los miembros de este grupo hacia los valles orientales para acceder a productos agrícolas. Durante el viaje se realizan ceremonias para propiciar su buen término, actuando especialmente las representaciones de camélidos (confeccionadas algunas en piedra, otras en grasa del vientre de llama o en harina de maíz blanco), en aquellas dedicadas a los mallkus, cerros con los que se topan las caravanas a su paso (Nielsen, 1997).

El control de estos objetos, como forma de control mágico-religioso de enfermedades y para fines propiciatorios, se observa también entre los kallawaya de La Paz, Bolivia (Gea, 1997; Madrid, 2000-2001).

En todos estos casos, la confección y el uso de las illas nos hablan de la particular noción de lo tecnológico en el mundo andino, al entender la producción y el producir como el acto de «criar la vida» (van Kessel, 1989), reproduciendo actos 
de las divinidades; proceso en el que actúa la «magia simpática» (Madrid, 20002001), aquella por la cual se controla lo representado en la cultura material.

\section{ANTECEDENTES EN LA ARQUEOLOGÍA DE ALTA MONTAÑA}

A continuación, haremos una breve reseña de los hallazgos en montaña, tanto de estatuillas como de cuerpos humanos, para conocer la magnitud de la práctica ritual incaica en el área centro-sur andina.

Hasta la fecha, en la cordillera de los Andes se han registrado 192 montañas con restos arqueológicos, de las cuales el $96 \%$ se localizan al sur de Cuzco (Vitry, 2008).

En estos contextos se encontraron 27 cuerpos humanos, 17 (63\%) de sexo femenino y 10 (37\%) de sexo masculino (Vitry, 2008). El número de estatuillas es más elusivo, ya que se han perdido muchas de ellas debido a los saqueos.

Los primeros hallazgos en las altas cumbres andinas comienzan a finales del siglo XIX y los realizan viajeros naturalistas, buscadores de tesoros, mineros y aficionados al montañismo.

Fueron principalmente hallazgos fortuitos que en su mayoría incluyeron estatuillas antropomorfas y zoomorfas (Aldunate del Solar, 2001), atados de troncos (Ceruti, 1997), ratones (Díaz Costa, 1966; Le Paige, 1978; Millán de Palavecino, 1966), vasijas cerámicas y tiestos, textiles (Beorchia Nigris, 1985) y seres humanos, en el marco de la ceremonia incaica de capacocha (Duviols, 1976; Mc Ewan \& Van de Guchte, 1993; Niles, 1993; Ceruti, 1997, 2003; D'Altroy, 2003; Reinhard \& Ceruti, 2000; 2010; Besom, 2009; 2013).

Cabe remarcar que las miniaturas antropomorfas y zoomorfas han sido encontradas en variedad de sitios, además de las cimas de las montañas.

La diversidad de ambientes involucra desde los costeros, la Isla de la Plata en Ecuador, por ejemplo, hasta los fondos de valle, como los hallazgos realizados en el mismo centro de Cuzco, en Haucaypata y en Sacsayhuaman, sin olvidar los realizados bajo el Lago Titicaca (Dransart, 1995: 10-11), como se puede observar en el cuadro 1.

\section{LA CAPACOCHA INCAICA DEL VOLCÁN LLULLAILLACO. CONTEXTOS DE HALLAZGOS}

El volcán Llullaillaco (6739 msnm) (fig. 1) se encuentra entre el departamento de Los Andes de la provincia de Salta, Argentina, y la II Región de Chile, Antofagasta, en un medio árido conocido como Puna, una depresión geológica de 3800 m de altitud media, caracterizada por una gran aridez, vegetación dispersa, bajas precipitaciones, alta insolación y gran amplitud térmica durante el día (Cabrera, 1957). 
Análisis distribucional de las estatuillas incaicas encontradas en el volcán Llullaillaco

Cuadro 1 - Principales hallazgos de enterramientos incaicos

\begin{tabular}{|c|c|c|c|c|c|}
\hline Lugar & Año & $\begin{array}{c}\text { Altitud } \\
\text { (msnm) }\end{array}$ & $\begin{array}{c}\text { Restos } \\
\text { humanos }\end{array}$ & $\begin{array}{c}\text { Estatuillas } \\
\text { antropomorfas }\end{array}$ & $\begin{array}{l}\text { Estatuillas } \\
\text { zoomorfas }\end{array}$ \\
\hline Cerro Doña Ana, Coquimbo-Chile & 1870 & 5690 & - & - & $x$ \\
\hline Bahía de Drake, La Plata-Ecuador & 1892 & 0 & $X$ & $x$ & $x$ \\
\hline Cerro Chachani, Arequipa-Perú & 1896 & 6084 & $x$ & - & - \\
\hline Nevado de Chañi, Salta-Argentina & 1905 & 5896 & $x$ & - & - \\
\hline $\begin{array}{l}\text { Nevado de Chuscha, Salta- } \\
\text { Argentina }\end{array}$ & 1922 & 5500 & $x$ & - & - \\
\hline Walla Walla, Cuzco-Perú & 1937 & 4800 & $x$ & $x$ & $\mathrm{X}$ \\
\hline Cerro El Plomo, Santiago-Chile & 1954 & 5425 & $x$ & $x$ & $x$ \\
\hline Volcán Galán, Catamarca-Argentina & 1956 & 5900 & - & $x$ & - \\
\hline Volcán Pichu Pichu, Arequipa-Perú & $\begin{array}{c}1963, \\
1989, \\
1997\end{array}$ & 5600 & $x$ & $x$ & $X$ \\
\hline Cerro Esmeralda, Iquique-Chile & 1976 & 905 & $x$ & $x$ & - \\
\hline Cerro El Toro, San Juan-Argentina & 1964 & 6380 & $x$ & - & - \\
\hline $\begin{array}{l}\text { Cerro Aconcagua, Mendoza- } \\
\text { Argentina }\end{array}$ & 1985 & 5300 & $x$ & $x$ & $X$ \\
\hline Chokepukio, Cuzco-Perú & 1994 & 3138 & $x$ & $x$ & $X$ \\
\hline Volcán Sara Sara, Arequipa-Perú & 1996 & 5505 & $x$ & $x$ & $x$ \\
\hline Haucaypata, Cuzco-Perú & 1996 & 3400 & - & - & $x$ \\
\hline Cerro Huaracante, Arequipa-Perú & 1997 & 5200 & - & $x$ & $x$ \\
\hline Nevado de Ampato, Arequipa-Perú & 1997 & $\begin{array}{c}6312 y \\
5850\end{array}$ & $x$ & $x$ & $X$ \\
\hline Volcán Misti, Arequipa-Perú & 1998 & 5822 & $x$ & $x$ & $x$ \\
\hline Volcán Quéhuar, Salta-Argentina & 1999 & 6130 & $x$ & $x$ & $x$ \\
\hline Volcán Llullaillaco, Salta-Argentina & 1999 & 6715 & $x$ & $x$ & $x$ \\
\hline
\end{tabular}




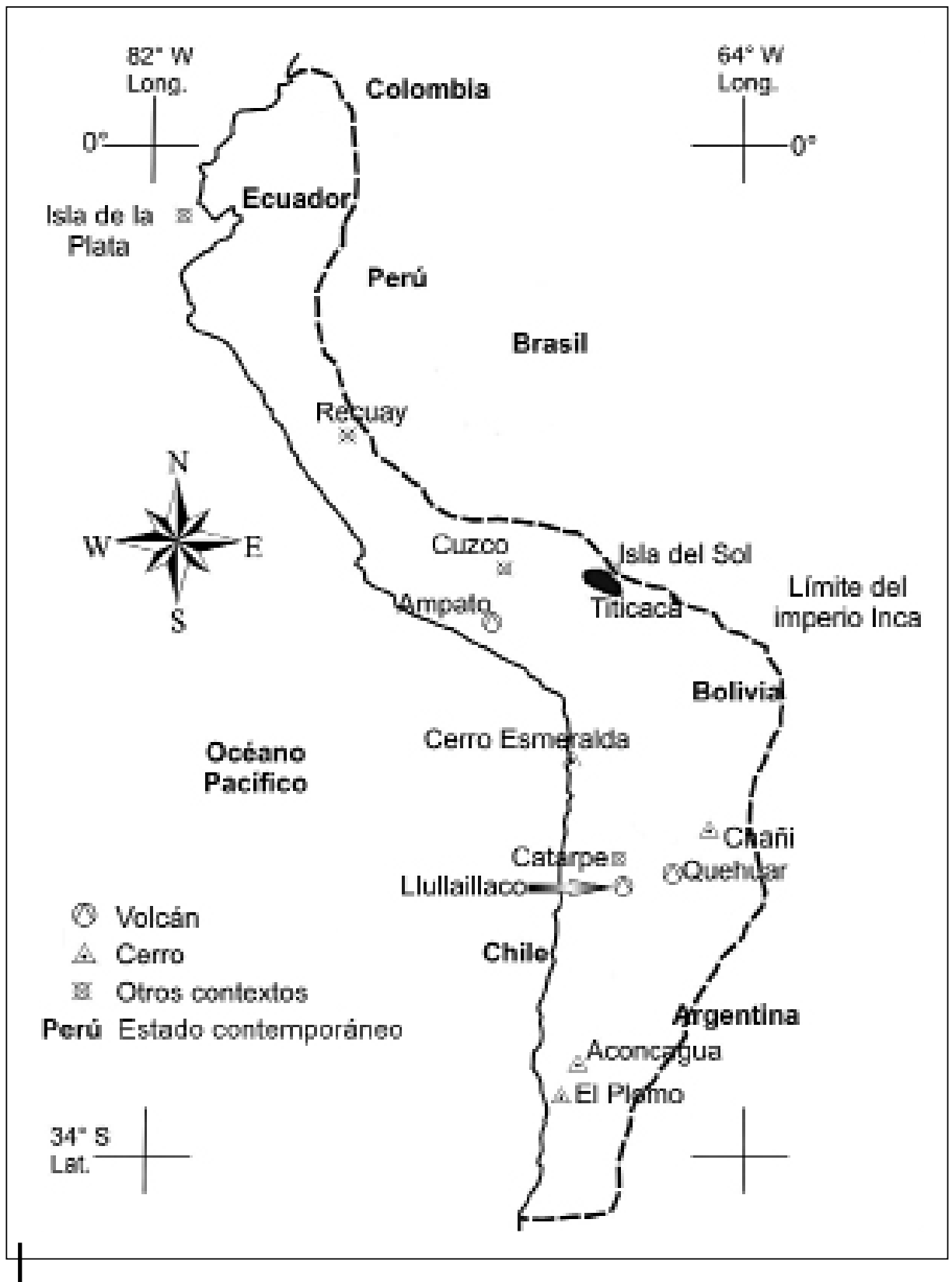

Figura 1 - Extensión del Tahuantinsuyu y ubicación de los sitios de capacocha más estudiados Redibujado de Bray et al., 2005: 87 
La plataforma donde fueron ofrecidos los tres niños del Llullaillaco se encuentra en un promontorio plano, a 20 m debajo de la cima. Dentro de ella se encontró un círculo de piedras redondeadas que podrían haber sido demarcaciones de las tumbas (Reinhard \& Ceruti, 2010: 66).

La expedición de 1999, dirigida por Reinhard, encontró tres entierros y un numeroso acompañamiento mortuorio (fig. 2), a profundidades comprendidas entre 1 y $2 \mathrm{~m}$, dentro de oquedades naturales del terreno sobre las que se formaron cámaras funerarias y luego fueron rellenadas con el sedimento (Reinhard \& Ceruti, 2000: 46-54).

Una de estas cámaras funerarias fue localizada en el sector suroeste de la plataforma. Medía $1 \mathrm{~m}$ de ancho y 1,7 $\mathrm{m}$ de profundidad. De ella fue recuperado el cuerpo de un niño de 7 años de edad (fig. 3). A su lado llevaba una bolsa cubierta con plumas que contenía hojas de coca, dos bolsas de piel que contenían cabellos humanos, dos pares de sandalias y dos huaracas.

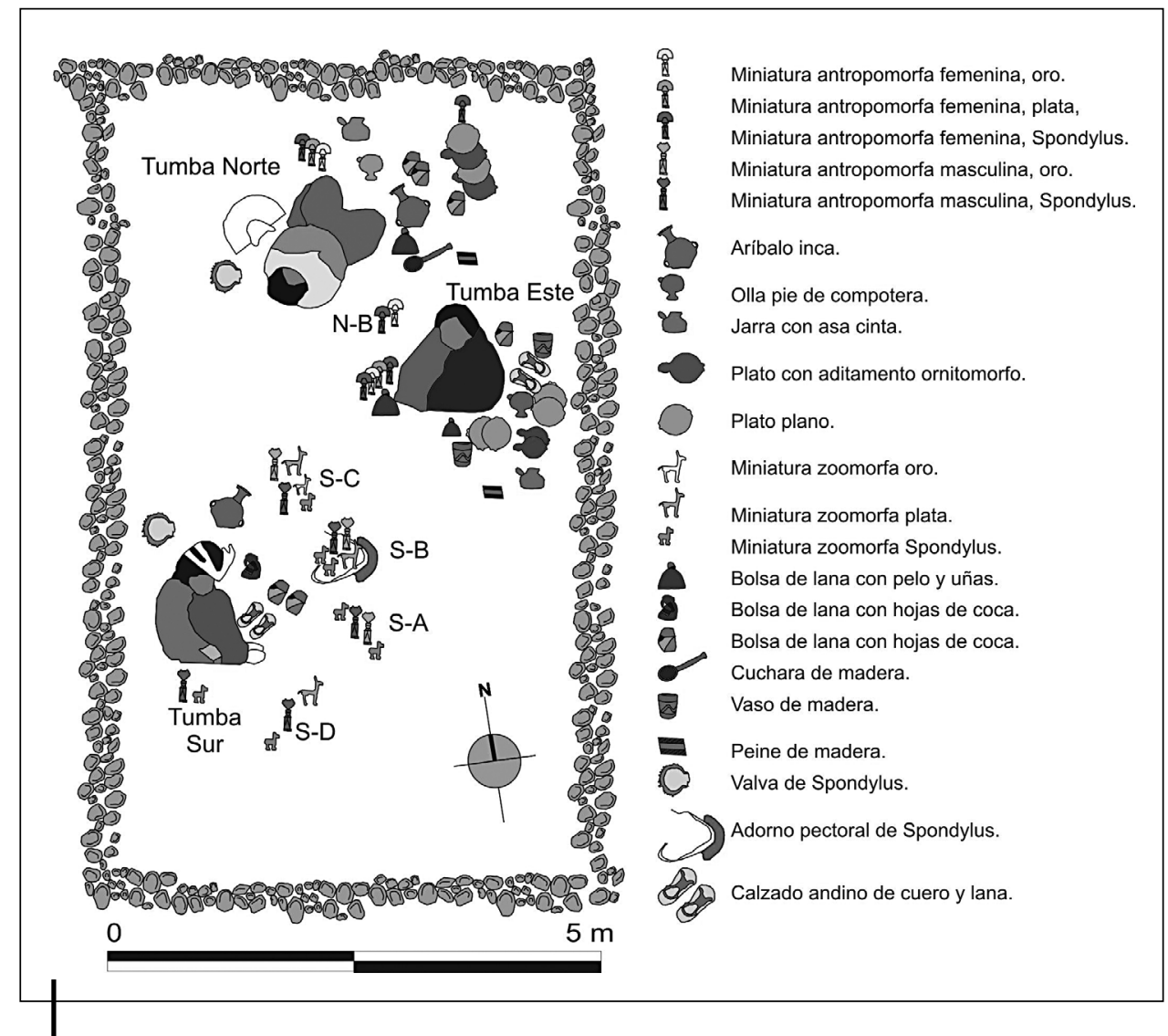

Figura 2 - Plano de planta de la plataforma ceremonial, con indicación de los contextos estudiados Redibujado del Catálogo del Museo de Arqueología de Alta Montaña de Salta, 2005: 44 

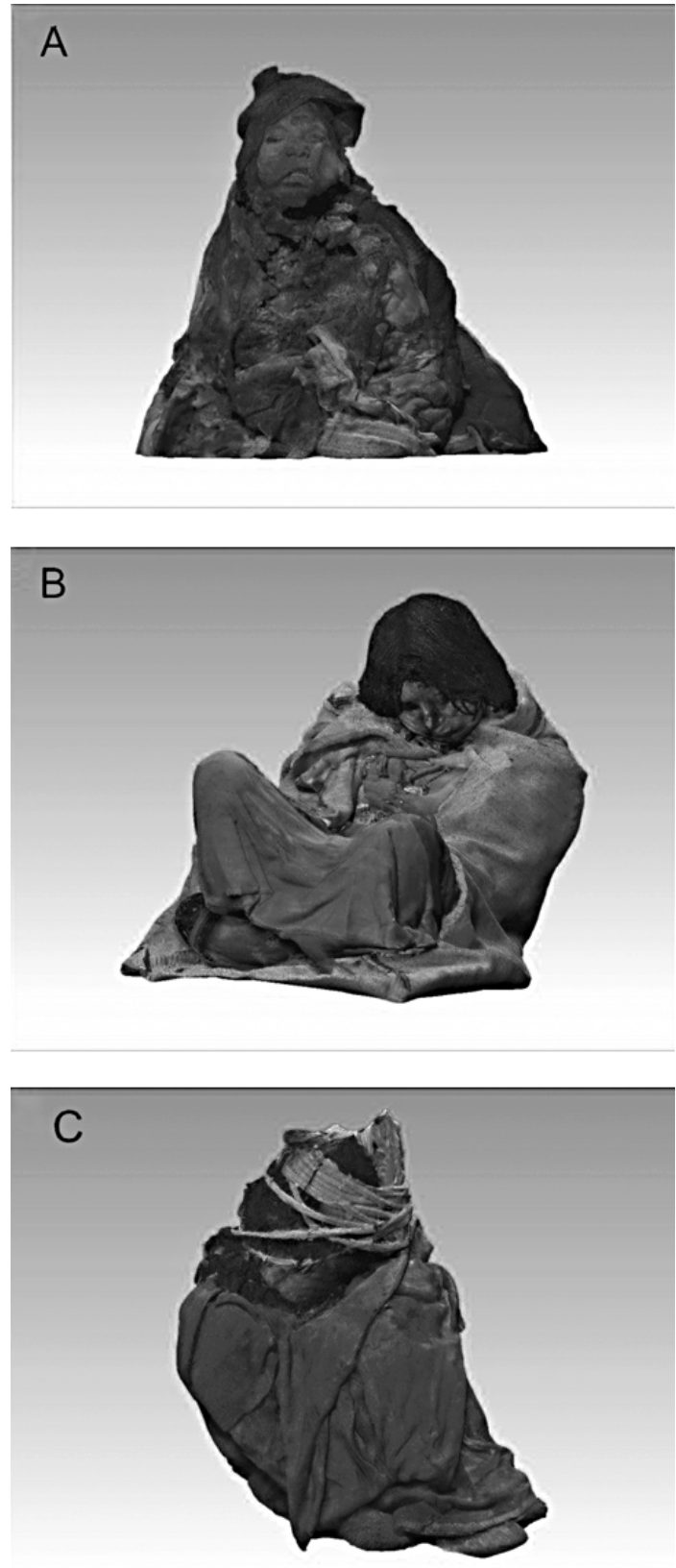

$$
1
$$

Figura 3 - Las ofrendas humanas del Llullaillaco. A: niña de 6 años, B: niña de 15 años, C: niño de 7 años

Imágenes cortesía del Museo de Arqueología de Alta Montaña, Salta 
A su derecha, fue encontrado un pequeño espacio cercado con piedras planas que encerraban dos miniaturas de Spondylus, una antropomorfa masculina y otra zoomorfa. También fueron encontradas una vasija aribaloide y una concha de Spondylus parcialmente trabajada, en el relleno de la tumba (Reinhard \& Ceruti, 2010: 72).

La tumba Este, por su parte, se encontró en el centro de la plataforma y contenía el cuerpo de una niña de 6 años, dentro de un espacio de $0,8 \mathrm{~m}$ de ancho y 1,75 m de profundidad. Su cuerpo presentaba signos de quemaduras por la acción de un rayo, al igual que las vestimentas y los objetos acompañantes.

Junto a ella fueron hallados artefactos cerámicos incas (un aríbalo, tres pares de platos, una olla con pedestal, dos cuencos y un jarro), un par de vasos de madera (kero), cuatro bolsas de lana conteniendo alimentos, una bolsa cubierta de plumas con hojas de coca, un par extra de mocasines y un par de sandalias y un conjunto de bolsas de piel conteniendo cabello humano. También fueron recuperadas cuatro miniaturas antropomorfas femeninas, una de oro, otra de plata y dos de Spondylus (Reinhard \& Ceruti, 2010: 77).

La tumba Norte, por otro lado, fue identificada en el sector septentrional de la plataforma y medía $1 \mathrm{~m}$ de ancho y más de $2 \mathrm{~m}$ de profundidad. En ella se depositó el cuerpo de una niña de 15 años, en posición decúbito dorsal. Alrededor suyo fueron colocados artefactos cerámicos y de madera, como un aríbalo, cuatro platos, un jarro, una olla con pedestal, dos kero, una cuchara y un peine. Llevaba también seis bolsas tejidas conteniendo alimentos, fajas de lana (mamachumpi) y dos bolsas pequeñas que contenían cabellos humanos. También se recuperaron tres miniaturas femeninas hechas de oro, plata y Spondylus (Ceruti, 2003: 80-81).

Suman 32 las miniaturas incas encontradas en las tumbas (fig. 4) y en los espacios de ofrenda cercanos. De ellas, quince son zoomorfas y representan a camélidos, de las cuales seis son metálicas (dos de oro y cuatro de plata), mientras que nueve son de mullu (Reinhard \& Ceruti, 2000; 2010; Ceruti, 2003; Mignone, 2009).

Las estatuillas antropomorfas masculinas suman once, cuatro de oro, una de plata (piezas macizas y huecas) y seis de Spondylus. Las estatuillas femeninas suman doce en total, cinco metálicas (cuatro de plata y una de oro) y siete de Spondylus. Las estatuillas antropomorfas, en su mayoría, tienen el rostro completamente visible, en donde se representaron ojos, nariz, boca y orejas perforadas (Ceruti, 2003). Su vestimenta es típicamente incaica y llevan como adornos penachos de plumas amarillas, blancas, rojas o negras según la miniatura (Ceruti, 2003).

\section{CONSIDERACIONES TEÓRICO-METODOLÓGICAS}

Un debate se ha dado en el seno de las investigaciones sobre los contextos arqueológicos del Llullaillaco para saber si las ofrendas encontradas en la cima del volcán fueron realizadas en un evento o en varios (Vitry, comunicación personal, 2014). 

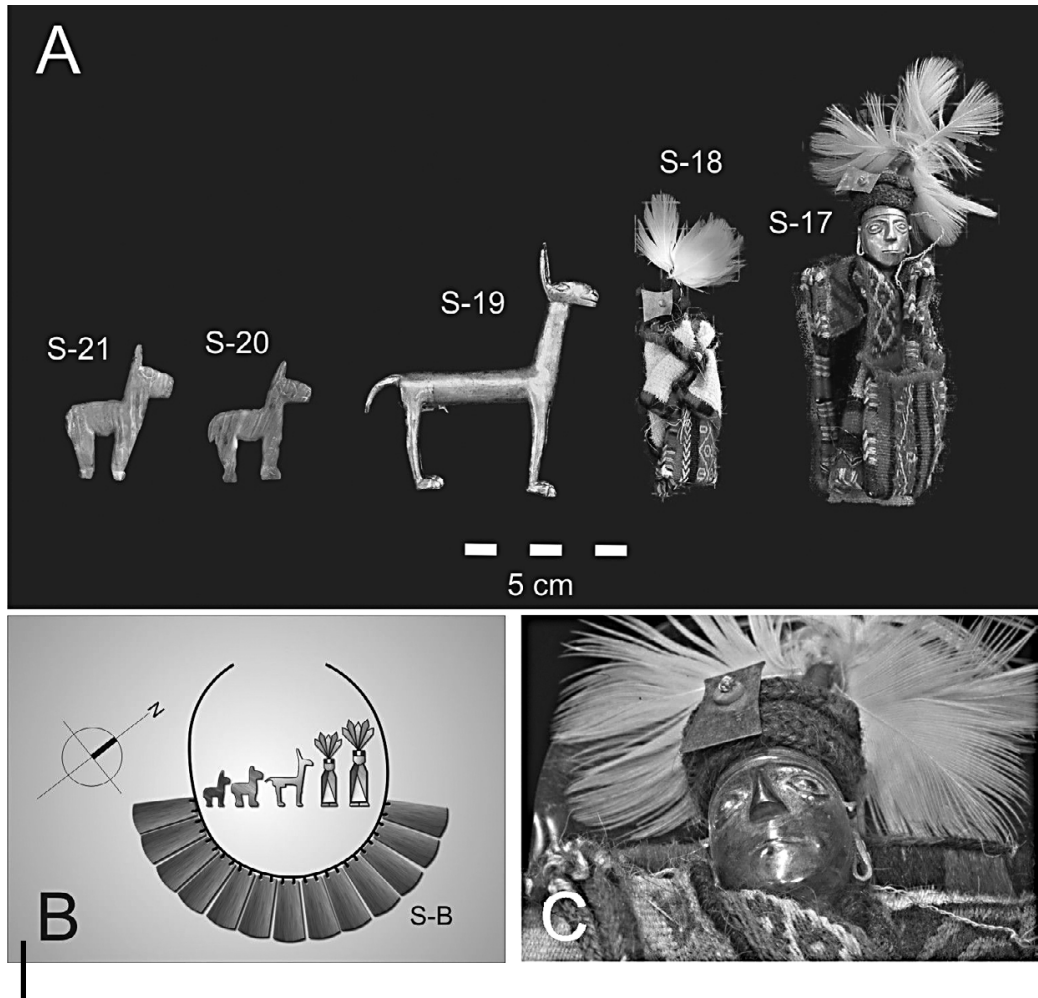

Figura 4 - Detalles de estatuillas antropomorfas y contextos de hallazgos: contexto S-C (A), contexto S-B (B), estatuilla antropomorfa masculina de oro (C) (S-17) Según Reinhard \& Ceruti, 2000: 162

Imágenes cortesía del Museo de Arqueología de Alta Montaña, Salta

Si bien las excavaciones desarrolladas en 1999 por el equipo internacional que desenterró las ofrendas fueron registradas de forma minuciosa en cuanto a la distribución y profundidad de los objetos, lo relativo al proceso de formación del sitio que permitiría una reconstrucción de las secuencias de depositación por sucesiones estratigráficas no es claro y permanece aún sin poder dilucidarse.

Además, el énfasis en la tipología cuzqueña de los objetos que se observan en los antecedentes de investigación deja de lado las múltiples miradas que este particular contexto arqueológico permite tener.

Es por ello que empleamos herramientas de la arqueología cuantitativa para evaluar si en las asociaciones entre los objetos, y no en su tipología únicamente, existen posibilidades de interpretación que aporten al estado actual de conocimiento sobre el tema.

Siguiendo a Shennan (1991) y a Carlson (2012), nuestra labor tuvo como objetivo mostrar las formas en las que se traduce un problema arqueológico en términos estadísticos y las cuestiones metodológicas asociadas a esta traducción. 
El empleo de métodos cuantitativos en la Arqueología implica el estudio de objetos, excavaciones y datos arqueológicos procedentes de los informes y memorias, antes que de los estudios basados en el trabajo de laboratorio, tales como propiedades del suelo, análisis químicos, etc.

Por lo tanto, los métodos cuantitativos han de ser considerados no como una especialidad científica diferenciada dentro de la Arqueología, como el análisis de polen, por ejemplo, o las variadas técnicas de caracterización de artefactos, sino como parte del arsenal de útiles, destinado a la identificación de una regularidad (Orton, 1980), de la estructuración y las relaciones de los objetos en el registro arqueológico.

Debido a que las matemáticas constituyen un sistema abstracto de relaciones, existe, pues, la posibilidad de que estas nos ayuden en la tarea de reconocer un esquema en el registro arqueológico y de especificar su naturaleza.

Si bien los enfoques cuantitativos han sido duramente criticados por la corriente posprocesualista, que hace hincapié en la interpretación del registro arqueológico y en su contexto de significación (Johnson, 2000: 136), muchas veces debemos recurrir a una base de cuantificación ya que el estudio de las relaciones entre fenómenos sigue siendo fundamental y, en muchos casos, la cuantificación es la forma más útil de investigar la estructura relacional del registro arqueológico.

Dentro de las estrategias cuantitativas, elegimos la estadística, cuyo rol en la exploración, clarificación e identificación de relaciones espaciales es de suma importancia, sin dejar de lado la necesidad de complementar la investigación cuantitativa con la interpretación del registro (para una interpretación etnohistórica de los contextos arqueológicos del Llullaillaco, véase Mignone, 2015).

La escala de análisis en la que trabajaremos es, según la arqueología espacial, la microescala (Clarke, 1977: 11), empleando el software R Studio Desktop 0.99.484 para el cálculo estadístico, debido a su superioridad con respecto a los paquetes estadísticos y SIG utilizados comúnmente (Connolly \& Lake, 2007: 136), a la simplicidad conceptual, en la ejecución y en la matemática, aspectos favorecidos por la bibliografía que los desarrolladores y contribuidores han hecho accesible y cuya oferta crece constantemente (Baxter, 2015: 2).

Empleamos herramientas de la estadística no paramétrica que permiten realizar inferencias exploratorias en muestras que, como la analizada, son pequeñas y no responden a la hipótesis de normalidad, por lo que se conoce poco acerca de los parámetros de la población mayor (Connolly \& Lake, 2007: 122; Shennan, 1991; Baxter, 2015: 212).

Dentro de la estadística no paramétrica, el test de Kruskal-Wallis es una alternativa al ANOVA, mientras que el test de dos muestras de Wilcoxon lo es para el $t$ de Student. Estos dos métodos son comúnmente usados por arqueólogos y han probado ser herramientas cuantitativas robustas, útiles y comparativamente fáciles de computar e interpretar (vanPool \& Leonard, 2011). 


\section{RESULTADOS}

Los objetos recuperados del volcán Llullaillaco fueron clasificados en siete grupos (cuadro 2).

Los objetos de uso personal, como las bolsas que contienen uñas, pelos y alimento, así como la vestimenta y los accesorios (calzados, collares, penachos y vinchas), fueron depositados a corta distancia de los cuerpos en las tumbas (de 0 a $0,68 \mathrm{~m}$ ), mientras que los objetos suntuarios, como las miniaturas antropomorfas y zoomorfas, se encuentran mayoritariamente en espacios de ofrendas fuera de ellas y a distancias mayores que el intervalo anterior (fig. 5).

Antes de realizar el estudio de estadística inferencial, quisimos saber si la muestra seguía una distribución normal. El resultado del test de normalidad ShapiroWilk, $p<0,05$ (2,134319e-12), permite rechazar la hipótesis de normalidad de la muestra.

Debido a estos resultados realizamos dos pruebas no paramétricas: $\chi^{2}$ de KruskalWallis y test de Wilcoxon.

La prueba de Kruskal-Wallis indica que existen diferencias significativas $(p<0,05$ $[5,391$ e-15]) en las varianzas de la variable distancia, con base en la especificidad sexual del objeto (su pertenencia al mundo de lo femenino o de lo masculino). Por su parte, el test de Wilcoxon fue aplicado para examinar la distribución de dos variables (distancia y profundidad) para considerar si pueden provenir de una sola población (Sinopoli, 1991: 207).

Como muestra el cuadro 3, la distancia de los objetos a los cuerpos es, según la prueba de regresión logística binaria multivariante, el factor que mejor explica la diferencia de distribuciones entre las illas (cuadro 3).

Cuadro 2 - Porcentajes de los tipos de ofrenda

\begin{tabular}{|l|c|}
\hline \multicolumn{1}{|c|}{ Tipo de ofrenda } & $\%$ \\
\hline Ofrenda animal (Anim.off) & 0,03 \\
\hline Bolsa-Chuspa (Bag) & 16,3 \\
\hline Vestimenta y accesorios (D/A/Footw) & 18,3 \\
\hline Miniaturas antropomorfas (Illa.ant) & 21,15 \\
\hline Miniaturas zoomorfas (Illa.zoo) & 14,4 \\
\hline Cuerpos humanos (Mummy) & 2,9 \\
\hline Vasijas de cerámica, vasos de madera y cucharas (Ware) & 24 \\
\hline
\end{tabular}


Análisis distribucional de las estatuillas incaicas encontradas en el volcán Llullaillaco

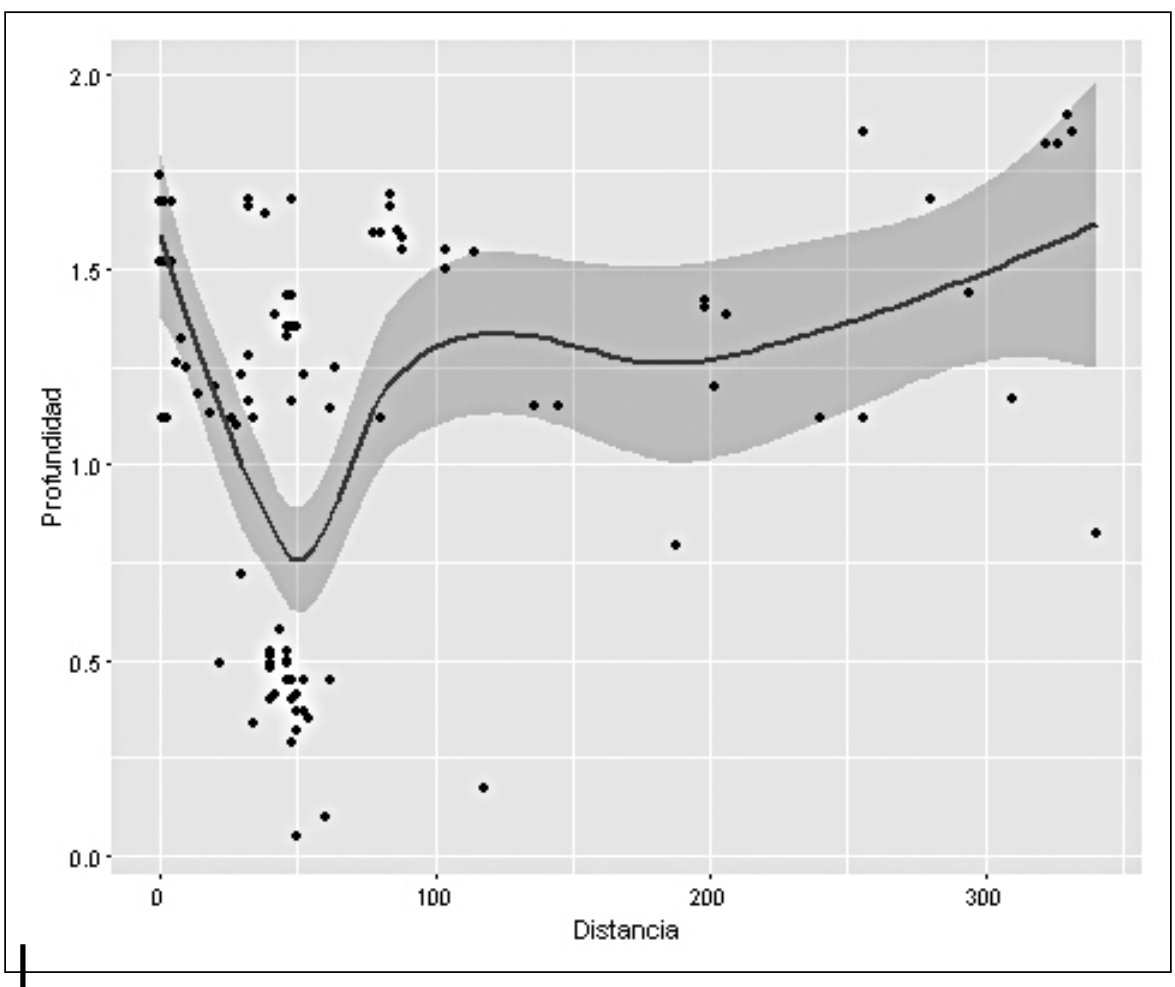

Figura 5 - Distribución de frecuencias absolutas de objetos según la distancia y la profundidad Elaboración: P. Mignone

Cuadro 3 - Coeficientes y factores de la regresión logística

\begin{tabular}{|c|c|c|c|c|c|}
\hline \multicolumn{2}{|c|}{ Coeficientes } & Estimación & $\begin{array}{c}\text { Error } \\
\text { estándar }\end{array}$ & Valor Z & $\operatorname{Pr}(>|z|)$ \\
\hline \multicolumn{2}{|c|}{ Intercepción } & $-0,62$ & 1,24 & $-0,50$ & 0,61 \\
\hline \multicolumn{2}{|r|}{ Diámetro } & $-1,94$ & 1,32 & $-1,40$ & 0,14 \\
\hline \multirow{4}{*}{$\begin{array}{l}\frac{\pi}{U} \\
\frac{\pi}{\pi} \\
\frac{\pi}{0} \\
0\end{array}$} & $(0,68-1,36)$ & 2,31 & 1,12 & 2 & $0,03 *$ \\
\hline & $(1,36-2,04)$ & 19,82 & 2635,32 & 0,008 & 0,99 \\
\hline & $(2,04-2,72)$ & 19,85 & 3250,27 & 0,006 & 0,99 \\
\hline & $(2,72-3,4)$ & 2,59 & 0,94 & 2,75 & $0,005^{* *}$ \\
\hline \multicolumn{2}{|c|}{ Profundidad } & 0,48 & 0,62 & 0,77 & 0,43 \\
\hline
\end{tabular}


Se observan dos valores p significativos. El primero se encuentra en el intervalo $0,68-1,36\left(^{*}\right)$, que señala los espacios internos de las tumbas, y el segundo muestra el intervalo 2,72-3,4 $\left(^{* *}\right)$, los valores más alejados de los cuerpos.

Esto sugiere que las tumbas y su contenido, por un lado, y los espacios de ofrendas con las miniaturas, por otro, pertenecen a contextos diferentes.

Es por ello que comparamos la distribución de objetos con respecto a los cuerpos mediante una prueba de Wilcoxon.

La comparación entre la tumba Norte, donde fue encontrada la joven de 15 años, y los espacios de ofrenda cercanos (N-A, N-B, N-C, N-D y N-E, según Ceruti, 2003) muestra que no hay un efecto significativo atribuido al grupo $(W=89$; $Z=-1,0489 ; p>0,05[0,3223] ; r=19,901)$ en cuanto a la profundidad de los objetos, pero sí en lo que respecta a la variable distancia $(W=33,5 ; Z=-3,1798$; $p>0,05[0,0002441] ; r=7,490828)$. Esto significa que los objetos se ubican en la tumba en relación al cuerpo, mientras que los espacios de ofrenda cercanos no se distribuyen con relación a él.

Este hecho se observó también en la tumba Sur (correspondiente al lugar de entierro del «niño»). La tumba y los espacios de ofrenda cercanos ( $\mathrm{S}$, según Ceruti, 2003) pertenecen al mismo grupo $(W=44 ; Z=-1,4689 ; p>0,05[0,1514]$; $r=9,838699$ ), en cuanto a la profundidad de los objetos, pero el efecto de grupo es significativo en lo que respecta a la distancia $(W=247 ; Z=3,1817 ; p<0,05$ [0,0002441]; $r=74,12565)$.

La relación entre el niño y la joven en cuanto a su distribución, muestra que las diferencias de grupo son significativas $(p<0,05)$ en ambas variables.

El mismo fenómeno se observa entre el contenido de las tumbas Sur y Este (del niño y de la niña de seis años, respectivamente).

Las tumbas de ambas niñas, por último, presentan diferencias significativas por efecto de grupo en la variable profundidad $(W=128 ; Z=-4,1689 ; p<0,05$ $[2,496 \mathrm{e}-06] ; r=28,62167)$, pero no en la variable distancia $(W=488 ; Z=0,0456$; $p>0,05[0,9687] ; r=109,1201)$.

Tomando las tumbas aisladamente, las niñas pertenecen a un mismo grupo en cuanto a la distancia que mantienen los objetos con los cuerpos, mientras que el niño no puede ser agrupado con el mismo criterio dentro del conjunto. Pertenece, en términos de distancia, a otro contexto.

A su vez, los espacios de ofrenda que se ubican fuera de las tumbas del niño y la doncella (la niña del rayo no posee ninguno cerca), S y $\mathrm{N}$ respectivamente, pertenecen a un mismo grupo en términos de profundidad $(W=130 ; Z=-1,1531$; $p>0,05[0,2734] ; r=29,06888)$, mientras que la prueba aplicada sobre la variable distancia muestra, por el contrario, una diferencia significativa por efecto del grupo $(W=67 ; Z=-2,6215 ; p<0,05$ [0,005859]; $r=14,98166)$, indicando que no pertenecen al mismo contexto. 


\section{CONCLUSIÓN}

Basándonos en la distancia de los objetos a las momias, tenemos entonces, como resultado, cuatro conjuntos distintos:

1. Tumbas Norte y Este, de las niñas.

2. Tumba Sur, del niño.

3. Conjuntos de ofrendas S.

4. Conjuntos de ofrendas $\mathrm{N}$.

Los conjuntos de ofrendas, formados predominantemente por miniaturas antropomorfas y zoomorfas, no guardan relación espacial con los cuerpos.

A su vez, las niñas forman parte de la misma lógica de inhumación, mientras que el niño no forma parte del grupo. Esto podría indicar criterios o preceptos diferenciales para el entierro de niños y niñas, por un lado, y de ofrendas y cuerpos, por el otro.

La segregación entre objetos y cuerpos se puede deber también a distintos momentos de entierro, en lugar de tratarse de una sola inhumación.

Por último, un próximo estudio etnohistórico podría indicar la significancia cultural de la diferencia entre las estatuillas antropomorfas, con vestimenta y adornos claramente incaicos (Abal, 2010), y los niños, que por el contrario, llevan vestimenta, peinados y adornos propios de sus lugares de origen (cuenca del Titicaca, según Abal, 2010: 229-236).

Proponemos hasta el desarrollo de nuevas investigaciones, que la distancia entre los objetos y cuerpos humanos podría ser también un reflejo de la diferencia de estatus entre las comunidades de pertenencia de los niños y los funcionarios del Estado incaico, encargados de la conquista y pacificación de este sector de los Andes Meridionales.

\section{Agradecimientos}

Agradezco al CONICET por las sucesivas becas que permitieron el desarrollo de mis investigaciones. A Leonardo Bellomo, quien dibujó la distribución de los conjuntos de ofrendas; a la directora del Museo de Arqueología de Alta Montaña de Salta, Lic. Gabriela Recagno, por facilitar las instalaciones del museo y la colección arqueológica para mis estudios; a las doctoras María Ester Albeck y Sara Mata, por acompañar con su dirección mis investigaciones posdoctorales. 


\section{Referencias citadas}

ABAL, C., 2010 - Arte textil incaico en ofrendatorios de la alta cordillera andina: Aconcagua, Llullaillaco, Chuscha, 444 pp.; Buenos Aires: Fundación CEPPA.

ALDUNATE DEL SOLAR, C., 2001 - The inka in Tarapacá and Atacama. In: In the footsteps of the Inka in Chile (C. Aldunate del Solar \& L. Cornejo, eds.): 18-33; Santiago de Chile: Museo Chileno de Arte Precolombino.

ARRIAGA, J. de, 1968 [1621] - The Extirpation of Idolatry in Peru, 192 pp.; Lexington: University of Kentucky Press.

BAXTER, M., 2015 - Notes on Quantitative Archaeology and R, xii + 276 pp.; Disponible en http://www.mikemetrics.com/\#/book-quantitative-archaeolog/4568129078 (consulta realizada el 7 de septiembre de 2015).

BEORCHIA NIGRIS, A., 1985 - El enigma de los santuarios indígenas de Alta Montaña, 410 pp.; San Juan: Universidad Nacional de San Juan.

BESOM, T., 2009 - Of Summits and Sacrifice. An Ethnohistoric Study of Inka Religious Practices, xii +230 pp.; Texas: University of Texas Press.

BESOM, T., 2013 - Inka Human Sacrifice and Mountain Worship: Strategies for Empire Unification, 329 pp.; Albuquerque: University of New Mexico Press.

BETANZOS, J. de., 1987 [1551-1557] - Suma y narración de los Incas, xciii + 317 pp.; Madrid: Atlas.

BRAY, T., MINC, L., CERUTI, M. C., CHÁVEZ, J. A., PEREA, R. \& REINHARD, J., 2005 A compositional analysis of pottery vessels associated with the Inca ritual of capacocha. Journal of Anthropological Archaeology, 24 (1): 82-100.

CABRERA, Á., 1957 - La vegetación de la Puna Argentina. Revista de Investigaciones Agrícolas, XI (4): 317-512; Buenos Aires.

CARLSON, D., 2012 - An R Companion to Quantifying Archaeology by Stephen Shennan, 43 pp.; Texas: A\&M University, Anthropology Department. Disponible en http://people.tamu.edu/ dcarlson/quant/Shennan/

CERUTI, M. C., 1997 - Arqueología de Alta Montaña, 110 pp.; Salta: Editorial Milor.

CERUTI, M. C., 2003 - Llullaillaco: sacrificios y ofrendas en un santuario inca de Alta Montaña, 342 pp.; Salta: Editorial de la Universidad Católica de Salta (EUCASA).

CLARKE, D., 1977 - Spatial Archaeology, xi + 386 pp.; Londres: Academic Press.

COBO, B., 1964 [1653] - Historia del Nuevo Mundo, tomos XVI y XCII; Madrid: Ediciones Atlas.

CONNOLLY, J. \& LAKE, M., 2007 - Geographical Information systems in Archaeology, xx + 338 pp.; Cambridge: Cambridge University Press.

D'ALTROY, T., 2003 - Los Incas, 425 pp.; Barcelona: Ariel.

DÍAZ COSTA, R., 1966 - Prácticas religiosas en el incanato, en relación con la "momia" del cerro El Toro. In: La "momia" del cerro El Toro. Investigaciones arqueológicas en la Cordillera de la provincia de San Juan República Argentina (J. Schobinger, ed.): 124167; Mendoza: Editorial Fasanella.

DRANSART, P., 1995 - Elemental Meanings: Symbolic Expression in Inka Miniature Figurines. Research Papers, 40: 1-58; Londres: Institute of Latin American Studies, University of London.

DUVIOLS, P., 1976 - La Capacocha. Mecanismo y función del sacrificio humano. Su proyección geométrica. Su papel en la política integracionista y en la economía redistributiva del Tahuantinsuyu. Allpanchis, 9: 11-57. 
DUVIOLS, P., 1986 - Cultura Andina y Represión. Procesos y visitas de idolatrías y hechicerías. Cajatambo siglo XVII, Ixxxvi + 568 pp.; Cuzco: Centro de Estudios Rurales Andinos "Bartolomé de las Casas".

FARRINGTON, I., 1998 - The Concept of Cusco. Tawantinsuyu, 5: 53-59.

FLORES OCHOA, J. A., 1974 - Enqa, Enqaychu illa y Khuya Rumi: aspectos mágicoreligiosos entre pastores. Journal de la Société des Américanistes, 63: 245-262.

GEA, H., 1997 - Los kallawayas talladores de amuletos y talismanes. Kallawaya. Publicación del Instituto de Investigaciones en Antropología Médica y Nutricional, 4: 61-64.

GONZÁLEZ HOLGUÍN, D., 1989 [1613] - Vocabulario de la lengua general de todo el Perú Ilamada lengua Qquichua o del Inca, 707 pp.; Lima: Universidad Nacional Mayor de San Marcos.

HERNÁNDEZ PRÍNCIPE, R., 1923 [1622] - Mitología Andina. Revista Inca, I (1): 25-78; Lima: Museo de Antropología de la Universidad Nacional Mayor de San Marcos.

JOHNSON, M., 2000 - Teoría Arqueológica, una introducción, 284 pp.; Barcelona: Editorial Ariel.

LE PAIGE, G., 1978 - Vestigios arqueológicos incaicos en las cumbres de la zona atacameña. Estudios Atacameños, 6: 37-48.

LÓPEZ, M., 2012 - Miniaturas andinas como imágenes materiales del bienestar, la fertilidad y la abundancia en Jujuy, Argentina. Estudios Avanzados, 18: 47-74.

MacCORMACK, S., 2000 - Processions for the Inca: Andean and Christian Ideas of Human Sacrifice, Communion and Embodiment in Early Colonial Peru. Archive fur Religionsgeschichte, 2 (1): 110-140; Berlín: De Gruyter.

MADRID, L., 2000-2001 - Señorío Kallawaya, médicos herbolarios. Aquellos talladores de amuletos y talismanes. Una aproximación a su interpretación. Kallawaya. Publicación del Instituto de Investigaciones en Antropología Médica y Nutricional, 7-8: 77-114.

MANZO, A. A. A., 2008 - De la extirpación de las idolatrías, 660 pp.; Buenos Aires: Editorial Dunken.

MANZO, A. A. A. \& RAVIÑA, M. G., 1996 - Augustukuy: Rito de multiplicación de los rebaños. Ceremonias rurales y su articulación con el registro arqueológico. Publicaciones de Arqueología, 48: 1-53.

Mc EWAN, C. \& VAN DE GUCHTE, M., 1993 - El tiempo ancestral y el espacio sagrado en el ritual estatal incaico. In: La antigua América: el arte de los parajes sagrados (R. Towsend, ed.): 379-371; Chicago: The Art Institute of Chicago.

MIGNONE, P., 2009 - Miniaturas zoomorfas del volcán Llullaillaco y contraste entre régimen estatal y vida comunitaria en la capacocha. Boletín del Museo Chileno de Arte Precolombino, 14 (1): 55-68.

MIGNONE, P., 2015 - Illas y Allicac: La capacocha del Llullaillaco y los mecanismos de ascenso social de los "Inkas de privilegio". Boletín del Museo Chileno de Arte Precolombino, 20 (2): 69-87.

MILLÁN DE PALAVECINO, M. D., 1966 - Elementos culturales asociados a la "momia" del cerro El Toro: piezas de vestimenta. Anales de Arqueología y Etnología, 21: 81-100.

MILLS, K., 1997 - Idolatry and Its Enemies: Colonial Andean Religion and Extirpation, 16401750, xii + 337 pp.; Princeton: Princeton University Press.

MURÚA, M. de, 1946 [1590] - Historia del Orígen y Genealogía Real de los Reyes Incas del Perú, xv + 444 pp.; Madrid: Consejo Superior de Investigaciones Científicas, Instituto Santo Toribio de Mogrovejo. 
MUSEO DE ARQUEOLOGÍA DE ALTA MONTAÑA, 2005 - Catálogo; Salta: Crivelli.

NIELSEN, A., 1997 - El tráfico caravanero visto desde La Jara. Estudios Atacameños, 14: 339-371.

NILES, S., 1993 - La arquitectura incaica y el paisaje sagrado. In: La antigua América: el arte de los parajes sagrados (R. Towsend, ed.): 347-373; Chicago: The Art Institute of Chicago.

ORTON, C., 1980 - Matemáticas para arqueólogos, 257 pp.; Madrid: Alianza Editorial

PIZARRO, P., 1986 [1571] - Relación del descubrimiento y conquista de los reinos del Perú (G. Lohmann Villena, ed.), cviii + 277 pp.; Lima: Pontificia Universidad Católica del Perú.

POLO DE ONDEGARDO, J., 1916 [1571] - Informaciones Acerca de la Religión y Gobierno de los Incas, vol. III, 2da. parte, 204 pp.; Lima: Sanmarti y Cia.

REBITSCH, M., 1957 - Die silbernen Gotter des Cerro Gallan, 207 pp.; Múnich: Nymphenburger Verlagsbuchhandlung.

REINHARD, J. \& CERUTI, C., 2000 - Investigaciones arqueológicas en el volcán Llullaillaco. Complejo ceremonial incaico de alta montaña, 217 pp.; Salta: Ediciones Universidad Católica de Salta (EUCASA).

REINHARD, J. \& CERUTI, C., 2010 - Inca rituals and sacred mountains: a study of the world's highest archaeological sites, xvi + 264 pp.; Los Ángeles: Cotsen Institute of Archaeology Press, University of California.

SALAZAR, L. \& R. BURGER, 2004 - Catalogue. In: Machu Picchu: Unveiling the Mystery of the Incas (R. Burger \& L. Salazar, eds.): 121-125; New Haven, Londres: Yale University Press.

SCHOBINGER, J., 1999 - Los santuarios de altura incaicos y el Aconcagua: aspectos generales interpretativos. Relaciones de la Sociedad Argentina de Antropología, XXIV: 7-27.

SCHOBINGER, J., 2001 - Los santuarios de altura y el Aconcagua: aspectos generales e interpretativos. In: El santuario incaico del cerro Aconcagua (J. Schobinger, ed.): 415-435; Mendoza: La Editorial de la Universidad Nacional de Cuyo (Ediunc).

SHENNAN, S., 1991 - Arqueología cuantitativa, 360 pp.; Barcelona: Crítica.

SINOPOLI, C., 1991 - Approaches to archaeological ceramics, 237 pp.; Nueva York: Springer Science+Business Media.

VAN KESSEL, J., 1989 - Ritual de producción y discurso tecnológico. Chungará. Revista de Antropología Chilena, 23: 73-91.

VanPOOL, T. L. \& LEONARD, R. D., 2011 - Quantitative Analysis in Archaeology, 345 pp.; Chichester, West Sussex, Malden: Wiley-Blackwell.

VITRY, C., 2008 - Los espacios rituales en las montañas donde los inkas practicaron sacrificios humanos. In: Paisagens Culturais. Contrastes sul-americanos (C. Terra \& R. Andrade, eds.): 47-65; Río de Janeiro: Escola de Belas Artes, Universidade Federal de Rio de Janeiro.

ZUIDEMA, T., 1973 - Kinship and Ancestor cult in three peruvian communities: Hernández Príncipe Account of 1622. Bulletin de I'Institut Français d'Études Andines, 2 (1): 16-33. 